ARTICLE

\title{
Possible absence of critical thickness and size effect in ultrathin perovskite ferroelectric films
}

\author{
Peng Gao 1,2,3,4, Zhangyuan Zhang ${ }^{1,5}$, Mingqiang Li1,4, Ryo Ishikawa ${ }^{6}$, Bin Feng ${ }^{6}$, Heng-Jui Liu , Yen-Lin Huang ${ }^{8}$, \\ Naoya Shibata ${ }^{6}$, Xiumei Ma1', Shulin Chen', Jingmin Zhang ${ }^{1}$, Kaihui Liu²,3,4, En-Ge Wang ${ }^{2,9}$, Dapeng Yu²,10, \\ Lei Liao ${ }^{5}$, Ying-Hao Chu ${ }^{8,11} \&$ Yuichi Ikuhara $6,12,13$
}

\begin{abstract}
Although the size effect in ferroelectric thin films has been known for long time, the underlying mechanism is not yet fully understood and whether or not there is a critical thickness below which the ferroelectricity vanishes is still under debate. Here, we directly measure the thickness-dependent polarization in ultrathin $\mathrm{PbZr}_{0.2} \mathrm{Ti}_{0.8} \mathrm{O}_{3}$ films via quantitative annular bright field imaging. We find that the polarization is significantly suppressed for films $<10$-unit cells thick $(\sim 4 \mathrm{~nm})$. However, approximately the polarization never vanishes. The residual polarization is $\sim 16 \mu \mathrm{Ccm}^{-2}(\sim 17 \%)$ at 1.5-unit cells $(\sim 0.6 \mathrm{~nm})$ thick film on bare $\mathrm{SrTiO}_{3}$ and $\sim 22 \mu \mathrm{Ccm}{ }^{-2}$ at 2-unit cells thick film on $\mathrm{SrTiO}_{3}$ with $\mathrm{SrRuO}_{3}$ electrode. The residual polarization in these ultrathin films is mainly attributed to the robust covalent $\mathrm{Pb}-\mathrm{O}$ bond. Our atomic study provides new insights into mechanistic understanding of nanoscale ferroelectricity and the size effects.
\end{abstract}

\footnotetext{
${ }^{1}$ Electron Microscopy Laboratory, School of Physics, Peking University, Beijing 100871, China. ${ }^{2}$ Collaborative Innovation Centre of Quantum Matter, Beijing 100871, China. ${ }^{3}$ Centre for Nanochemistry, College of Chemistry and Molecular Engineering, Peking University, Beijing 100871, China. ${ }^{4}$ Academy for Advanced Interdisciplinary Studies, Peking University, Beijing 100871, China. ${ }^{5}$ Department of Physics and Key Laboratory of Artificial Micro- and Nanostructures of Ministry of Education, Wuhan University, Wuhan 430072, China. ${ }^{6}$ Institute of Engineering Innovation, The University of Tokyo, Tokyo 113-8656, Japan. ${ }^{7}$ Department of Materials Science and Engineering, National Chung Hsing University, Taichung, Taiwan 40227, China. ${ }^{8}$ Department of Materials Science and Engineering, National Chiao Tung University, Hsinchu, Taiwan 30010, China. ${ }^{9}$ International Centre for Quantum Materials, School of Physics, Peking University, Beijing 100871, China. ${ }^{10}$ Department of Physics, South University of Science and Technology of China, Shenzhen 518055, China. ${ }^{11}$ Institute of Physics, Academia Sinica, Taipei, Taiwan 105, China. ${ }^{12}$ Nanostructures Research Laboratory, Japan Fine Ceramic Centre, Nagoya 456-8587, Japan. ${ }^{13}$ WPI Advanced Institute for Materials Research, Tohoku University, Sendai 980-8577, Japan. Correspondence and requests for materials should be addressed to P.G. (email: p-gao@pku.edu.cn) or to L.L. (email: liaolei@whu.edu.cn) or to Y.I. (email: ikuhara@sigma.t.u-tokyo.ac.jp).
} 
S table ferroelectricity in ultrathin films enables diverse nanoelectronic functions including high storage capacity of non-volatile ferroelectric memories. However, the existence of critical thickness below which the ferroelectricity disappears had been predicted long time ago due to the intrinsic size or depolarizing field effects ${ }^{-4}$. Experimentally observed critical thicknesses for epitaxial ferroelectric films usually are a few unit cells. For example, ferroelectricity in $\mathrm{PbTiO}_{3}$ (PTO) was observed in 10-unit cells thick film by scanning probe $e^{5}$ and 3-unit cells by synchrotron X-ray diffraction ${ }^{6}$. In $\mathrm{BaTiO}_{3}(\mathrm{BTO})$ films, the critical thickness has been reported to range from 7 -unit cells ${ }^{7}$ to 4 -unit cells ${ }^{8}$. For $\mathrm{BiFeO}_{3}$ (BFO), the ferroelectricity has been detected in 4 and 5 -unit cells thick films ${ }^{9,10}$. Some of these values are in good agreement with the theoretical work, for example, the critical thickness has been calculated to be 3-unit cells for PTO (ref. 11) and 6-unit cells for BTO (ref. 12), while other calculations suggested that the ferroelectricity might even exist in a single-unit cell thick pervoskite film with proper electrodes ${ }^{13}$, indicating the possible absence of a critical thickness. Furthermore, although the ferroelectricity does not disappear in the ultrathin films, the magnitude of polarization should be progressively decreased ${ }^{14-16}$ as the critical thickness is approached.

Despite intensive studies ${ }^{5-22}$, the possible presence of a critical thickness in ferroelectric thin films is debateable and the underlying physics of size effect is not yet fully understood because competing effects such as intrinsic size effect $^{1,2}$, surface properties ${ }^{2,23-25}$, substrate strain conditions ${ }^{26,27}$, interface chemistry and electrical boundary conditions ${ }^{13,16,28-32}$ contribute to the measured properties and thus complicate the behaviour. Early studies suggested that the intrinsic size effect existed in ferroelectrics and a critical volume (typically at hundreds of nanometres) was required to align the electric dipoles $^{1,2}$, while the later developments showed that the depolarizing field, which is caused by the incomplete compensation of polarization bound charges at interfaces, mainly account for the size effect ${ }^{3,4}$ and therefore the value of critical size should be much smaller than previous thought (at a few nanometres or tens of nanometres). The depolarizing field increases as the film thickness decreases, leading to the instability of ferroelectricity in the ultrathin films and fine particles'. Besides, the effects of interface and surface also become more pronounced when the film becomes thinner. For example, atomic displacements (ionic relaxation) in a conducting electrode ${ }^{28}$ or insulating substrate ${ }^{29}$, and strength of bonding ${ }^{32}$ have been found to be crucial for stabilizing the ferroelectricity in ultrathin films. The polarization of film could also be controlled by the vapour environments through ionic absorbate at the free surface $^{33}$, while the polarization underneath, on the other hand, in turn, strongly influence the surface structure ${ }^{25}$.

It is therefore crucial to quantitatively determine the individual effects of the substrate, surface, intrinsic size and their interactions to explore the nanoscale ferroelectricity in thin films. In fact, the recent advancements of aberration corrected (scanning) transmission electron microscopy [(S)TEM] have made it possible to measure the atomic displacements at the interface and surface in ferroelectric thin films ${ }^{31}$. For example, Chisholm et al. ${ }^{30}$ observed that the ferroelectric displacements even occurred in the electrode $\mathrm{SrRuO}_{3}$ (SRO) layer adjacent to the interface. Particularly, the atomically resolved annular bright-field (ABF) imaging 34 allows us to precisely quantify the localized polarization ${ }^{25}$ via robustly determining both cation and oxygen positions over a wide range of thickness. In contrast to the traditional electrical measurements that are not reliable for ultrathin films due to the leakage current issue $e^{5,15,16}$ or other indirect methods such as piezoelectric response ${ }^{10}$ and X-ray structural measurements ${ }^{6}$ which gather the response from the entire heterostructure (that is, surface, film and interface), the electron microscopy method enables us to precisely profile the structural parameters as well as the polarization throughout the thin film thus to isolate the roles from the surface $^{2,23-25}$, electrode-ferroelectric interface ${ }^{13,16,28-32}$, substrate strain $^{26,27}$ and other factors ${ }^{2-4}$. Nevertheless, systematic atomicscale electron microscopy studies of thickness-dependent polarization in ultrathin ferroelectric films have rarely been reported mainly due to the difficulties in high quality ultrathin film growth and TEM specimen preparation, and quantitative imaging and analysis.

Here, we study the thickness-dependent polarization in $\mathrm{PbZr}_{0.2} \mathrm{Ti}_{0.8} \mathrm{O}_{3}$ (PZT) films down to a single-unit cell thick via quantitative $A B F$ imaging in aberration corrected STEM. We find that the polarization $\left(\sim 96 \mu \mathrm{Ccm}^{-2}\right)$ in PZT on bare STO remains stable until the thickness becomes less than $\sim 10$-unit cells $(\sim 4 \mathrm{~nm})$, below which the polarization gradually decreases as the thickness decreases, for example, $\sim 81 \mu \mathrm{Ccm}^{-2}$ for 8.5-unit cells, $\sim 54 \mu \mathrm{Ccm}^{-2}$ for 6.5 -unit cells and $\sim 22 \mu \mathrm{Ccm}^{-2}$ for 4 -unit cells. Despite significant suppression, the polarization does not disappear even at 1.5-unit cells $\left(\sim 16 \mu \mathrm{Ccm}^{-2}\right)$, indicating the absence of critical thickness in this perovskite film. For a comparison, we also study the PZT thin films on STO substrate with SRO bottom electrode, in which the polarization is reduced to $\sim 22 \mu \mathrm{Ccm}^{-2}$ for 2 -unit cells. The atomic structure suggests that the residual polarization in the films below 3-unit cells is mainly attributed to the robust covalent $\mathrm{Pb}-\mathrm{O}$ bond. These results provide new insights into mechanistic understanding of the nanoscale polarization and size effect in ferroelectric thin films.

\section{Results}

PZT films on bare STO. Figure 1 shows the interface of a PZT thin film with thickness $\sim 28 \mathrm{~nm}$ on a STO substrate viewing along [010]. Judged by the contrast in the high angle annular dark field image in Fig. 1a, the $\mathrm{TiO}_{2}$ plane between the $\mathrm{SrO}$ and $\mathrm{PbO}$ planes is labelled as the interface ${ }^{29}$. Figure $1 \mathrm{~b}$ is the simultaneously recorded $\mathrm{ABF}$ image, from which both the cation columns $(\mathrm{Pb}$ and $\mathrm{Zr} / \mathrm{Ti})$ and oxygen columns are visible. The polarization in this sample is mainly upward ${ }^{30}$, that is, pointing towards right, because the $\mathrm{O}$ shifts to the left $\mathrm{Pb}$ column in Fig. 1b (ref. 25). The spontaneous polarization in each unit cell can be precisely calculated from: $P=\frac{e}{\Omega} \sum_{m}^{\mathrm{N}} Z_{m}^{*} \cdot \delta u_{m}$, where $e$ is the elementary charge, $\Omega$ is the volume of the unit cell, $Z_{m}^{*}$ is Born effective charge ${ }^{35}$ and $\delta u_{m}$ is the first-order change of the position vector of the $m$ th basis atom (in Fig. 1c). The maps in Fig. 1d-g are the measured lattice constants and bond lengths. These values are averaged along the interface and plotted in Fig. 1h-j. A transition zone of $\sim 3$-unit cells with suppressed polarization exists near the interface in the PZT film (so-called interfacial dead layer). Although the atomic displacements of $\mathrm{Pb}$ respective to $\mathrm{O}$ is much larger than that of Ti respective to $\mathrm{O}$ (ref. 36) in Fig. 1i,j, the Born effective charge for $\mathrm{Pb}$ is smaller ${ }^{35}$. As a result, the polarization contributed from $\mathrm{PbO}$ plane is close to that from $\mathrm{TiO}_{2}$ plane, as shown in Fig. $1 \mathrm{k}$. The total spontaneous polarization in PZT film is measured to be $\sim 96 \mu \mathrm{Ccm}^{-2}$ (see details in the 'Methods' section) and this measured value is representative (see Supplementary Fig. 1).

A few regions with various thicknesses are investigated (see details in the 'Methods' section). In a region with 8.5-unit cells thick in Supplementary Fig. 2, no significant difference than the bulklike region is observed. At 6.5-unit cells in Fig. 2a-c (see also Supplementary Fig. 3), the displacements of $\mathrm{Pb}$ and $\mathrm{Zr} / \mathrm{Ti}$ respective to $\mathrm{O}$ are reduced to $\sim 43 \mathrm{pm}$ and $\sim 19 \mathrm{pm}$ respectively, 


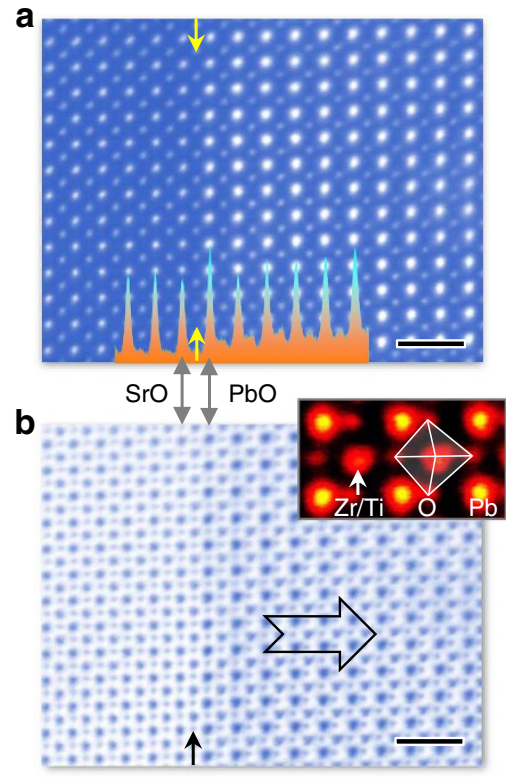

c

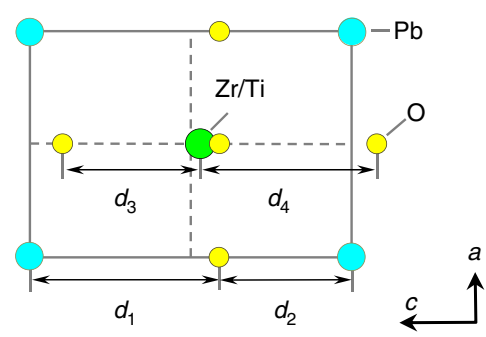

d

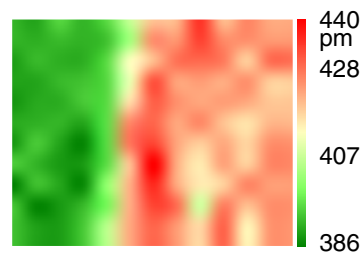

e

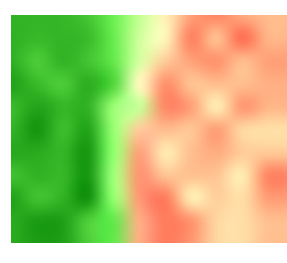

f

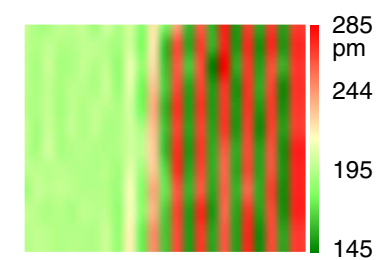

g

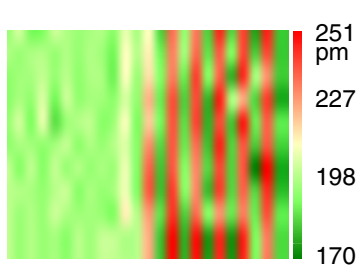

h

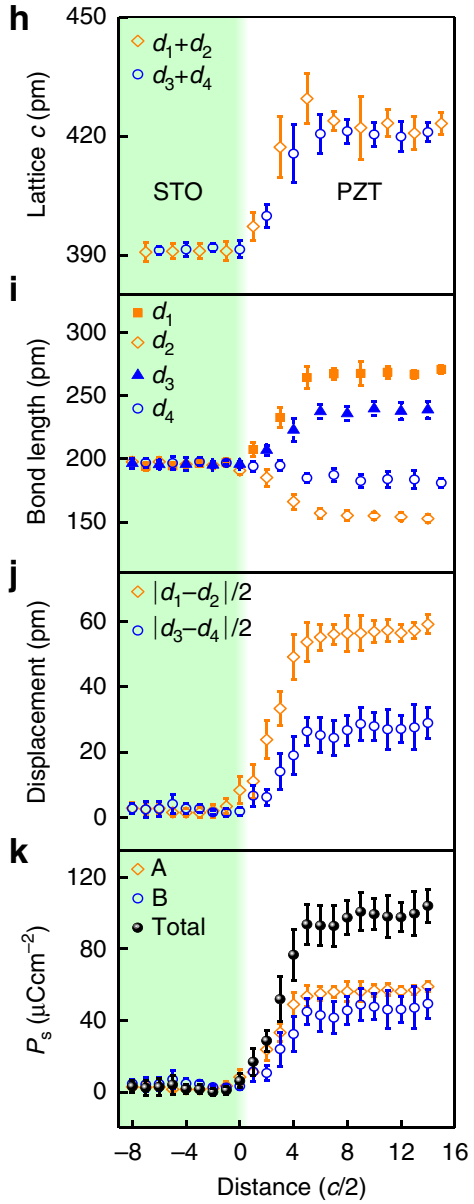

Figure 1 | Quantitative measurements of polarization in PZT thin film on SrTiO $\mathbf{3}_{\mathbf{3}}$ substrate. This region is 24-unit cells thick. (a) A high angle annular field image of a substrate-film interface. The arrow shows the position of interface, $\mathrm{TiO}_{2}$ plane, between the first $\mathrm{SrO}$ and $\mathrm{PbO}$ planes. Inset: the interface is determined by the intensity profile. Scale bar, $1 \mathrm{~nm}$. (b) The simultaneous recorded ABF image of the interface. Inset: enlarged view of PZT. The contrast is inverted for clarity. The polarization is upward, that is, pointing to the surface as denoted by the big arrow. Scale bar, $1 \mathrm{~nm}$. (c) Schematic shows the projection of tetragonal PZT seen along [010] direction. $d_{1}$ and $d_{2}$ denote the long and short distances between $\mathrm{Pb}$ atoms (cyan) and $\mathrm{O}$ atoms (yellow) along the $c$ direction; $d_{3}$ and $d_{4}$ denote the short and long distances between $\mathrm{Zr} / \mathrm{Ti}$ atoms (green) and $\mathrm{O}$ atoms (yellow) along the $c$ direction. Both $\mathrm{SrTiO}_{3}$ (STO) and PZT are expressed as $\mathrm{ABO}_{3}$ structure, where $\mathrm{A}$ represents for $\mathrm{Sr}$ and $\mathrm{Pb}$, and $\mathrm{B}$ represents for $\mathrm{Ti}$ or $\mathrm{Zr} / \mathrm{Ti}$. The lattice constants $\mathrm{c}$ is calculated from the (d) A sublattice, and (e) B sublattice. (f) The calculated bond lengths $d_{1}$ and $d_{2}$ in the AO plane. (g) The calculated bond lengths $d_{3}$ and $d_{4}$ in the $\mathrm{BO}_{2}$ plane. (h) Mean of lattice $c$. The error bar is the s.d. (i) Mean of bond lengths of $d_{1}, d_{2}, d_{3}$ and $d_{4}$. The error bar is the s.d. (j) Averaged displacements of $\left|d_{1}-d_{2}\right| / 2$ and $\left|d_{3}-d_{4}\right| / 2$. The error bar is the s.d. (k) Mean of polarization calculated from the displacements in $\mathrm{PbO}$ plane, $\mathrm{TiO}_{2}$ plane and the total.

The error bar is the s.d.

compared to $\sim 57 \mathrm{pm}$ and $\sim 28 \mathrm{pm}$ in Fig. 1, indicating the suppression of polarization. A dead layer $\sim 3$-unit cells still exists at the substrate-film interface. The width of interfacial dead layer is almost independent of the film thickness. However, no such transition zone with suppressed polarization is observed at the surface in this upward domain, which is in good agreement with previous observations ${ }^{25}$. Further reducing the thickness to 4 -unit cells in Fig. 2d-f (see also Supplementary Fig. 4), 2.5-unit cells in Supplementary Fig. 5, and 1.5-unit cells in Fig. 2g-i (see also Supplementary Fig. 6), the atomic displacements and polarization gradually decrease. However, they do not disappear even at 1.5-unit cells thick film in Fig. 2i, implying the polarization is suppressed but remains stable.

PZT films on STO with SRO electrode. We also performed similar analysis for ultrathin PZT film on STO substrate with SRO bottom electrode in Supplementary Fig. 7 to study the behaviour of thickness-dependent polarization. The ABF images and measured data from three thicknesses (8,4 and 2-unit cells) are shown in Fig. 3. Unlike the PZT film on bare STO with upward polarization, the polarization in PZT/SRO/STO is downward, pointing to the bottom interface, which is in good agreement with previous reports ${ }^{30,31}$. Besides the suppression of polarization at the bottom interface, the polarization also decreases at the top surface, which is consistent with previous studies of negatively poled PZT surface ${ }^{25,31}$. Similarly, in the thinner regions with thickness of 4-unit cells in Fig. 3d and 2-unit cells in Fig. 3g, the displacements (Fig. 3e,h) and the polarization (Fig. 3f,i) are also reduced. Still, they never disappear.

\section{Discussion}

The thicknesses-dependent polarization is summarized in Fig. 4a. Approximately, there are three regions: stable polarization zone I with film thickness $\geq 10$-unit cells, progressive decayed polarization zone II between 10 and 3-unit cells, and weak polarization zone III with film thickness $\leq 3$-unit cells. 
a

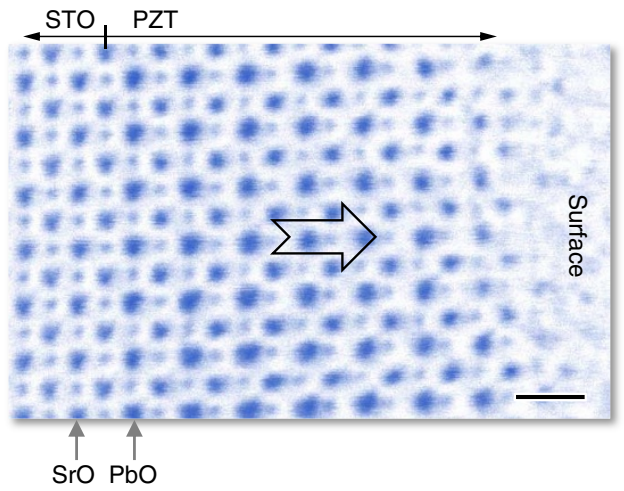

d

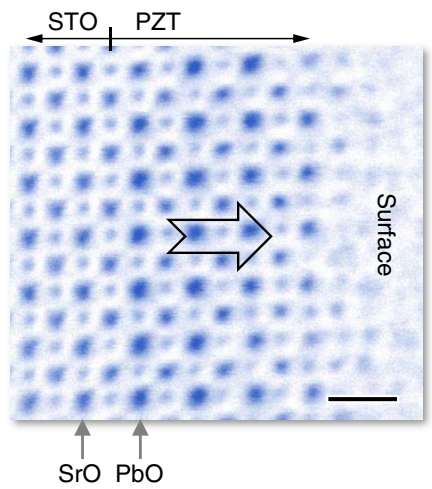

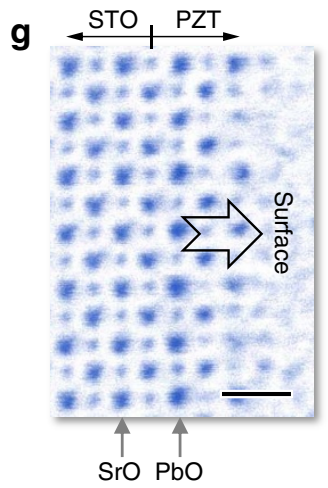
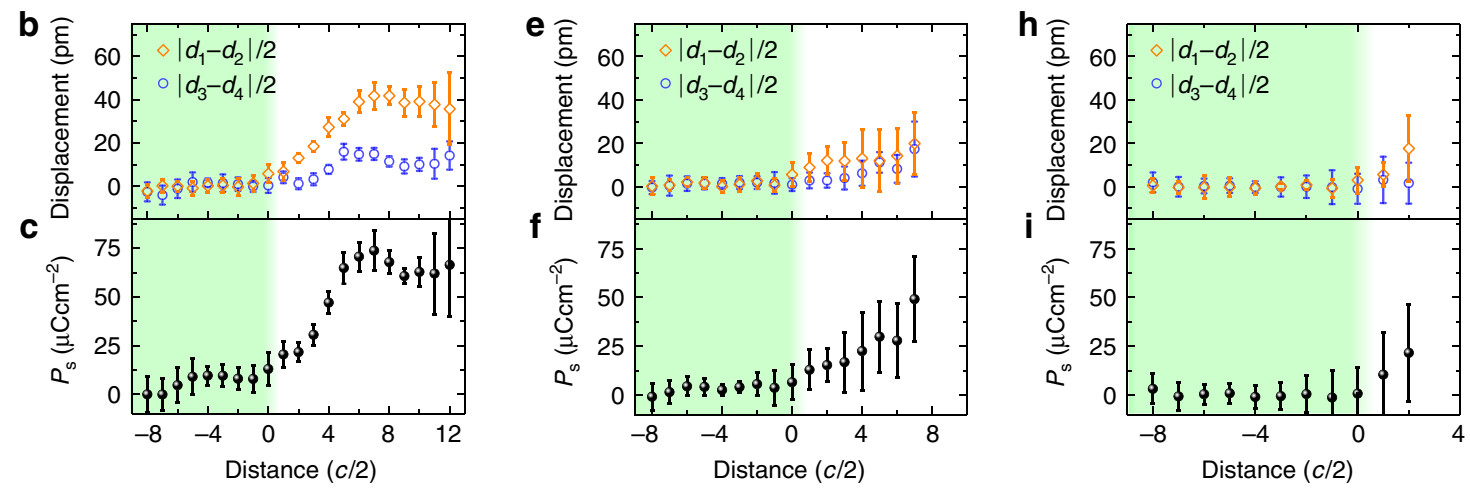

Figure 2 | Thickness-dependent atomic displacements in $\mathbf{P Z T}_{\mathbf{S}} \mathbf{S T T O}_{\mathbf{3}}$. (a) An ABF image from a region with thickness of 6.5-unit cells. Scale bar, $0.5 \mathrm{~nm}$. (b) Mean of displacements are calculated from of $\left|d_{1}-d_{2}\right| / 2$ and $\left|d_{3}-d_{4}\right| / 2$. The error bar is the s.d. (c) Mean of polarization calculated from the displacements. The error bar is the s.d. (d) An ABF image from a region with thickness of 4-unit cells. Scale bar, $0.5 \mathrm{~nm}$. (e) Mean of displacements are calculated from of $\left|d_{1}-d_{2}\right| / 2$ and $\left|d_{3}-d_{4}\right| / 2$. The error bar is the s.d. (f) Mean of polarization calculated from the displacements. The error bar is the s.d. (g) An ABF image from a region with thickness of 1.5 -unit cells. Scale bar, $0.5 \mathrm{~nm}$. (h) Mean of displacements are calculated from of $\left|d_{1}-d_{2}\right| / 2$ and $\left|d_{3}-d_{4}\right| / 2$. The error bar is the s.d. (i) Mean of polarization calculated from the displacements. The error bar is the s.d.

The polarization is upward in PZT on STO but downward with the SRO bottom electrode. As the thickness decreases, the magnitude of polarization is reduced. Interestingly, the orientation remains unchanged and no domain walls are observed in the ultrathin regions. The behaviour of thicknessdependent polarization magnitude in PZT films on both insulating STO and conducting SRO/STO is similar. In Fig. 4b, the measured polarization is normalized and compared with previous experimental and theoretical results. The presence of stable polarization in these ultrathin PZT films indicates that the polarization charges must be effectively compensated. The polarization screening can be from the external and/or internal charges ${ }^{37}$. The external screening is the charge compensation from the outside boundaries of ferroelectrics including both of top surface and bottom interface (that is, PZT/STO or PZT/SRO). The internal screening is determined by the distribution of free carriers or ionic charges and the associated structure changes within materials.

A rich variety of structures and properties were reported at the ferroelectric surface (refs 25,33 and references therein). Although all the surfaces in these TEM specimens unavoidably contain thin amorphous layers that were introduced during the TEM specimen preparation, the surface structure is dominated by the polarization underneath while less affected by the thin-surface amorphous layer ${ }^{25}$. In the case of upward polarization in the $\mathrm{PZT/STO}$, no significant surface reconstruction layer except the topmost atomic layer is observed for all the regions with different thickness in Fig. 2. This is in good agreement with previous experimental observations $s^{25}$. For the negatively poled surface in the PZT on SRO/STO, there is a thin reconstructed surface layer with reduced polarization, which was also reported before $^{31}$. Nevertheless, despite different internal structure relaxation in the vicinity of top surfaces between PZT/STO and PZT/SRO/STO TEM samples, the external boundary conditions at the top surfaces should be similar, as they were prepared with the same procedures and in the same environment.

Therefore, the polarization directions (that is, upward for PZT on STO in ref. 30 and reference therein, and downward for PZT on SRO/STO in refs 30,31) in these thin films should be governed by the boundary conditions of substrate/electrode. The width of interfacial deadlayer in PZT on SRO/STO is thinner than that in PZT/STO because the metallic SRO can provide negative charges to effectively screen the downward polarization at the interface and thus no significant internal structural relaxation within PZT is needed. For PZT on insulating STO substrate, formation of stripe domains ${ }^{6}$ or the atomic displacements in the insulating substrate ${ }^{29}$ were proposed to retain the stable polarization in ultrathin films. However, neither significant atomic displacements in the STO substrate nor $180^{\circ}$ stripe domains in PZT is observed in our sample (that is consistent with previous study ${ }^{30}$ and references therein), suggesting either positive charges provided by STO or the internal charges within the PZT thin film at the interface mainly act as the screening charge ${ }^{19}$.

For the PZT film on STO with a bottom electrode SRO, the depolarizing field can be estimated from the short-circuit conditions $E_{\mathrm{d}}=-2 \lambda_{\mathrm{eff}} P\left(d \varepsilon_{0}\right)^{-1}$ (refs 14,28,38), where $E_{\mathrm{d}}$ is the depolarizing field, and $\lambda_{\text {eff }}$ is the effective screening length, $\varepsilon_{0}$ is the permittivity of the free space, and $d$ is the thin film thickness. Given a constant $\lambda_{\text {eff }}=0.02 \mathrm{~nm}$ (refs $12,14,38,39$ and 
a

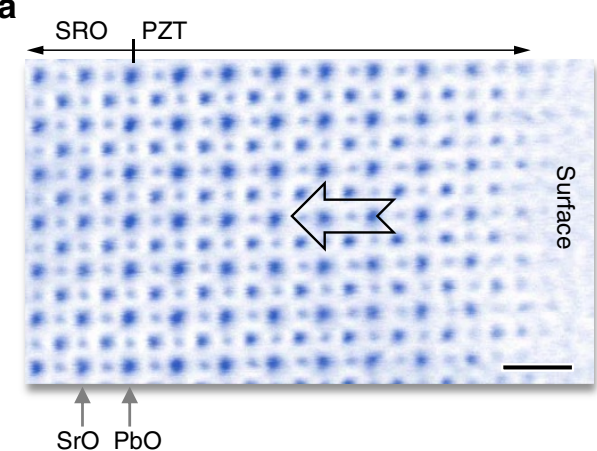

d

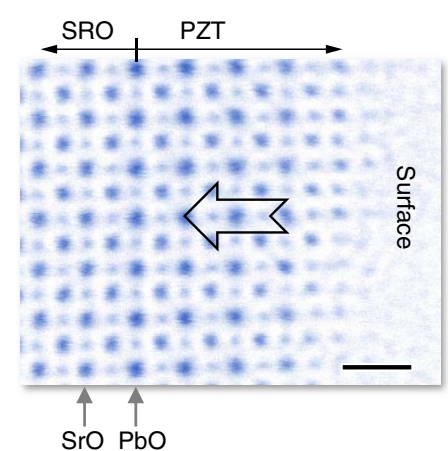

g

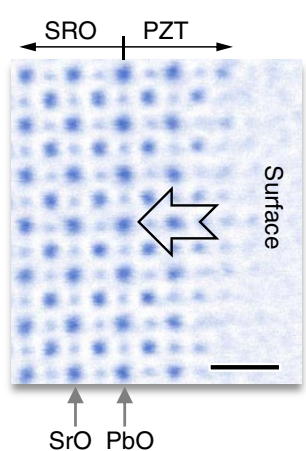

b

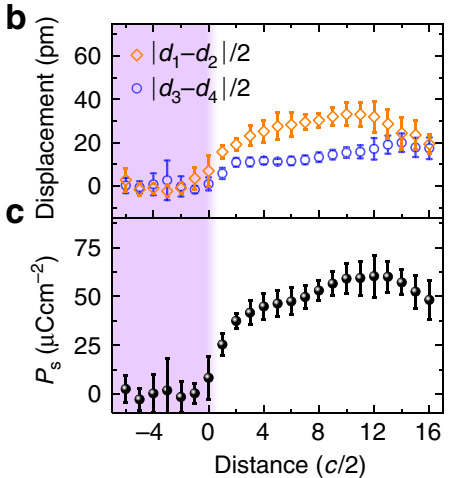

e

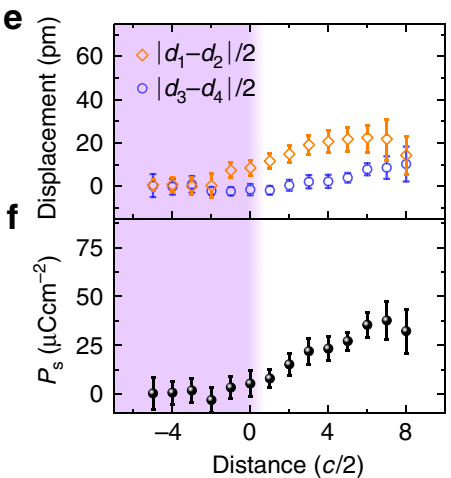

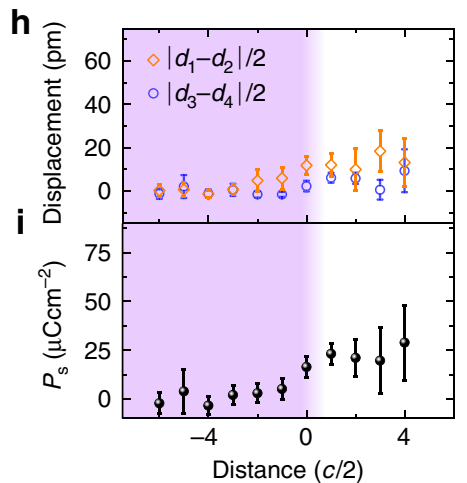

Figure 3 | Thickness-dependent atomic displacements in $\mathbf{P Z T / S r R u O} \mathbf{O}_{3} / \mathbf{S r T i O}_{3}$. (a) An ABF image from a region with thickness of 8-unit cells. Scale bar, $0.5 \mathrm{~nm}$. (b) Mean of displacements are calculated from of $\left|d_{1}-d_{2}\right| / 2$ and $\left|d_{3}-d_{4}\right| / 2$. The error bar is the s.d. (c) Mean of polarization calculated from the displacements. The error bar is the s.d. (d) An ABF image from a region with thickness of 4-unit cells. Scale bar, $0.5 \mathrm{~nm}$. (e) Mean of displacements are calculated from of $\left|d_{1}-d_{2}\right| / 2$ and $\left|d_{3}-d_{4}\right| / 2$. The error bar is the s.d. (f) Mean of polarization calculated from the displacements. The error bar is the s.d. (g) An ABF image from a region with thickness of 2-unit cells. Scale bar, $0.5 \mathrm{~nm}$. (h) Mean of displacements are calculated from of $\left|d_{1}-d_{2}\right| / 2$ and $\left|d_{3}-d_{4}\right| / 2$. The error bar is the s.d. (i) Mean of polarization calculated from the displacements. The error bar is the s.d.

references therein), the depolarizing field is approximately $0.7-1.2 \mathrm{Vnm}^{-1}$ (in Fig. 4c) for PZT/SRO/STO heterostructure. The estimated values are in good agreement with the density function theory calculations ${ }^{13}$ which showed depolarizing field of $0.7 \mathrm{Vnm}^{-1}$ for 2 -unit cells thick PTO film sandwiched between SRO electrodes. For the PZT film on bare STO, calculation of the depolarizing field is usually based on opencircuit conditions, under which both of the polarization and depolarizing field strongly depend on the concentration of internal charges ${ }^{40}$. In this case, the depolarizing field mainly occurs at the bottom interface and top surface while almost vanishes in the middle of film ${ }^{40}$.

When the thickness becomes as thin as 3-unit cells, the bottom interface zone (that is, the area subjected to the substrate-film interface effects) and the surface zone (that is, the area subjected to the surface effects) become overlapped and interact with each other and thus complicate the screening mechanism. In fact, X-ray diffraction experiment of stripe domain patterns ${ }^{6}$ at room temperature and theories of effective Hamiltonian ${ }^{11}$ and $a b$ initio calculation ${ }^{24}$ for $T=0 \mathrm{~K}$ suggested that 3 -unit cells is the critical thickness to retain the stable ferroelectricity in PTO films. In our study, when the thickness is $<3$-unit cells, the similar behaviour of suppressed polarization for both of the ultrathin PZT films on insulating substrate and metallic bottom electrode indicates that the compensation of polarization may be governed by the internal screening. Note both of the interface and surface contain ionic charges $25,30,33,39$ such as oxygen vacancies, $\mathrm{Pb}$ vacancies and absorbates. These defects may be even more easily introduced at the surface of TEM specimen foils during specimen preparation such as ion milling. In fact, the first principle calculations showed that the internal oxygen vacancies at the interface could effectively stabilize the polarization in ultrathin films ${ }^{30}$. Therefore, the small polarization can still survive because of the effective screening by these charged defects. Given polarization $\sim 16 \mu \mathrm{Ccm}^{-2}$ for 1.5 -unit cells thick PZT film on STO substrate, it requires 8 at.\% oxygen vacancy at the interface, that is, $(\mathrm{PbSr})(\mathrm{ZrTi}) \mathrm{O}_{2.92}$, if the polarization is fully compensated by the interfacial oxygen vacancies. For the PZT film on SRO/STO, the polarization screening may come from both of the ionic charges and bottom electrode compensation.

In Fig. 1k, we show that the total polarization is attributed to the atomic displacements in both $\mathrm{PbO}$ and $\mathrm{Zr} / \mathrm{TiO}_{2}$ planes. The displacements between $\mathrm{Zr} / \mathrm{Ti}$ and $\mathrm{O}$ approximately linearly decrease and almost disappear below 3-unit cells for PZT on $\mathrm{STO}$ in Fig. $4 \mathrm{~d}$. However, the $\mathrm{Pb}-\mathrm{O}$ displacements deviate from the linear decrease, instead they are even visible by naked eyes in the 1.5-unit cells region in Fig. 4e, where the arrows denote those oxygen columns shifting to the left $\mathrm{Pb}$ columns, similar to that in Fig. 1b. In the lead based ferroelectrics, the $\mathrm{Pb}-\mathrm{O}$ bonds through the hybridization of $\mathrm{Pb} 6 \mathrm{~s}$ and $\mathrm{O} 2 \mathrm{p}$ orbitals is covalent rather than pure ionic, and such covalent interaction accounts for the strong ferroelectric distortions ${ }^{36}$. Therefore, the residual polarization in the film below 3-unit cells is mainly contributed by the atomic displacements within the $\mathrm{PbO}$ planes due to the robust covalent $\mathrm{Pb}-\mathrm{O}$ bond, while the $\mathrm{Zr} / \mathrm{TiO}_{2}$ planes almost lose the polarization. Similar result for PZT on SRO/STO is shown in Supplementary Fig. 8.

In summary, we report a quantitative measurement of thickness-dependent polarization down to single-unit cell thick PZT films on STO substrate with and without bottom electrode. For the thick film ( $>10$-unit cells) on bare STO, the polarization 

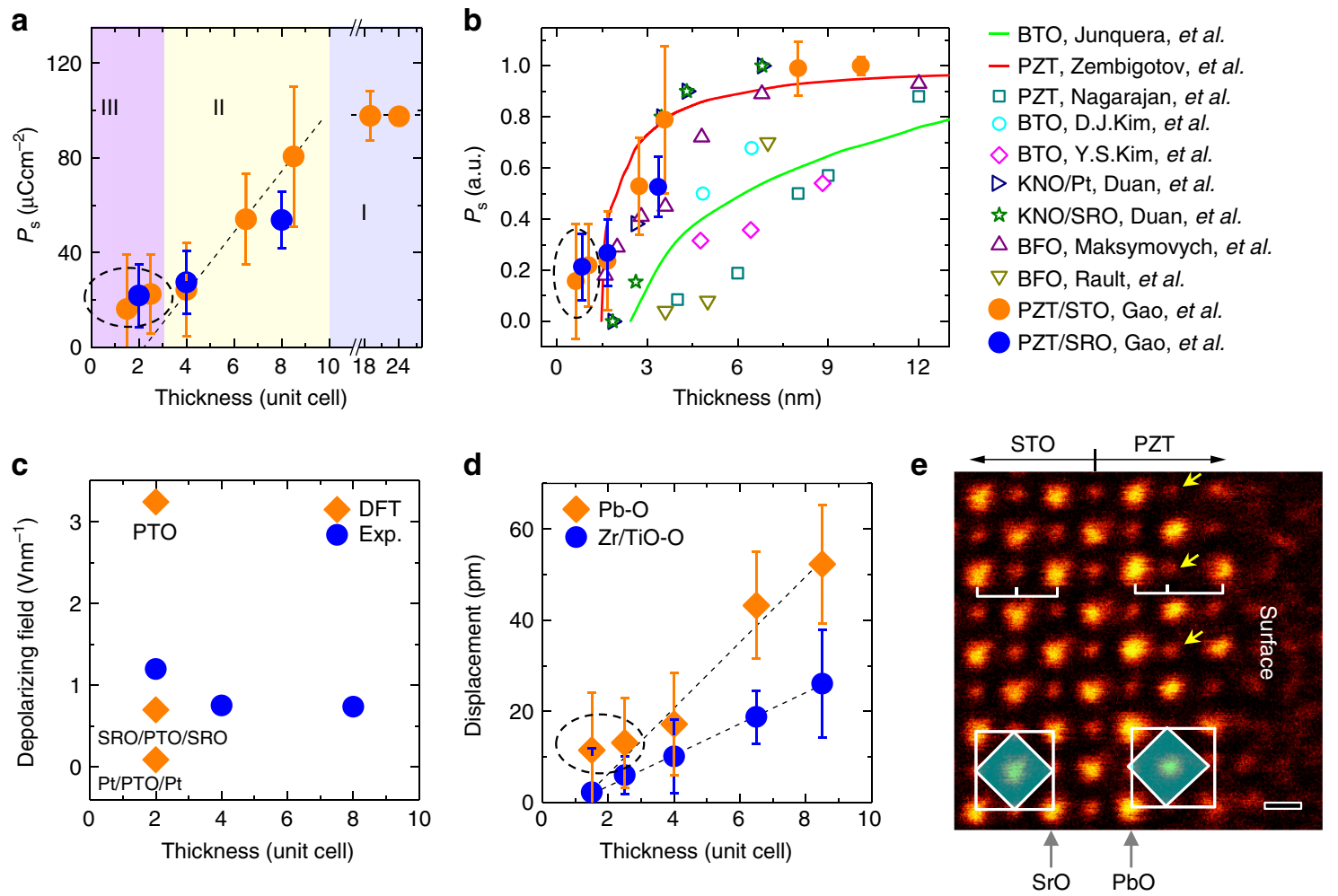

Figure 4 | Thickness-dependent polarization. (a) Calculated polarization is plotted as a function of the thickness. The error bar is the s.d. The dashed lines are plotted for eye guidance. (b) Comparison of the normalized polarization as a function of thickness with previous results. Solid red plot shows the predictions from mean-field Landau-type model for PZT from ref. 26. The solid green plot is first principle calculations for $\mathrm{SrRuO}_{3} / \mathrm{BaTiO}_{3} / \mathrm{SrRuO}_{3}$ (SRO/BTO/SRO) from ref. 12. The blue squares are experimentally measured values for SRO/PZT/SRO from ref. 16. The cyan circles are experimentally measured values for SRO/BTO/SRO from ref. 22. The magenta diamonds are experimentally measured values for SRO/BTO/SRO from ref. 15. The orange triangles are theoretical predictions for $\mathrm{Pt} / \mathrm{KNbO}_{3} / \mathrm{Pt}(\mathrm{Pt} / \mathrm{KNO} / \mathrm{Pt}$ ) from ref. 32. The dark green stars are theoretical predictions for SRO/KNO/SRO from ref. 32. The purple triangles are experimentally measured values for $\mathrm{BiFeO}_{3} / \mathrm{La}_{0.67} \mathrm{Sr}_{0.33} \mathrm{MnO}_{3} / \mathrm{SrTiO}_{3}$ (BFO/LSMO/STO) from ref. 10. The dark yellow triangles are experimentally measured values for BFO/LSMO/STO from ref. 21. The orange and blue solid discs are from this work. (c) Calculated depolarizing field is plotted as a function of the thickness for PZT on SRO/STO. The density function theory results from ref. 13 showing the depolarizing fields for free standing 2-unit cells thick $\mathrm{PbTiO}_{3}$ (PTO), 2-unit cells thick PTO sandwiched between SRO electrodes, and 2-unit cells thick PTO sandwiched between Pt electrodes. (d) Displacements of $\mathrm{Pb}$ respective to $\mathrm{O}$ and $\mathrm{Zr} / \mathrm{Ti}$ respective to $\mathrm{O}$ are plotted as a function of the thickness for PZT on STO. The error bars are the s.d. (e) An ABF image of 1.5-unit cells thick PZT on STO. The contrast has been inverted for clarity. The octahedron shifting to left in the PZT film is visible by the naked eye. The arrows showing the $\mathrm{O}$ are closer to the left $\mathrm{Pb}$, causing the atomic displacements and polarization.

Scale bar, $0.2 \mathrm{~nm}$.

is mainly upward. The suppression of polarization only occurs at the substrate-ferroelectric interface to form a ferroelectric dead layer with thickness $\sim 3$-unit cells, while at the top surface no significant surface structure except the topmost layer is observed. Between 10 and 3-unit cells, the polarization throughout the film is significantly suppressed, dominated by the thickness-dependent depolarizing field. Below 3-unit cells, the polarization is reduced but remains stable even for the PZT film on bare STO, for example, $\sim 22 \mu \mathrm{Ccm}^{-2}$ for 2.5 -unit cells and $\sim 16 \mu \mathrm{Ccm}^{-2}(\sim 17 \%)$ for 1.5 -unit cells. To best of our knowledge, the 1.5-unit cells thick PZT film is the thinnest perovskite film to experimentally show polarization so far. The observed polarization (or dipole moments) is stabilized by the soft phonon mode in ferroelectrics, which theoretically has a long wavelength. The analysed individual atomic terrace that is typically from 10 to 30 -unit cells in width (see also Supplementary Fig. 9) is likely enough to stabilize the dipole moments at these regions ${ }^{41}$. However, the effects of the thicker regions adjacent to the thinner region cannot be completely excluded. Further studies are needed to address such effect in future. With the bottom electrode SRO, the polarization of PZT film is mainly downward. The suppression of polarization occurs both at the ferroelectric-electrode interface and top surface. At 2-unit cells, the average polarization is reduced to $\sim 22 \mu \mathrm{Ccm}^{-2}$. The atomic structure shows that the robust covalent $\mathrm{Pb}-\mathrm{O}$ bonds mainly account for the residual polarization below 3-unit cells in the PZT films. Our study suggests that in practical thin films and devices the absence of critical thickness is possible due to the structural imperfection at the interface and surface.

\section{Methods}

Materials and characterization. Tetragonal PZT films oriented in the [001] direction were grown on (001) STO substrates with and without SRO bottom electrode by the pulsed laser deposition method using a $\mathrm{KrF}$ excimer laser $(\lambda=248 \mathrm{~nm})$. The laser beam was focused on the targets with an energy density of $\sim 2.5 \mathrm{Jcm}^{-2}$ and repetition rate of $10 \mathrm{~Hz}$. To grow the PZT thin film, the target with $5 \%$ excess lead was used to avoid the formation of lead deficiency in the film during the high temperature deposition. Growth was carried out at $650{ }^{\circ} \mathrm{C}$ and at oxygen pressure of 100 mTorr. In addition, for the sample with SRO bottom electrode, SRO was firstly deposited at $700{ }^{\circ} \mathrm{C}$ and 100 mTorr for obtaining higher quality thin film, and then cooled down to $650^{\circ} \mathrm{C}$ with the same oxygen atmosphere for subsequent growth of PZT thin film. All samples were finally postannealed at the same temperature and at oxygen pressures of $\sim 300$ Torr for $30 \mathrm{~min}$, and then cooled down to room temperature with a ramp rate of $5^{\circ} \mathrm{C} \mathrm{min}^{-1}$. The sample with SRO bottom electrode has been checked by piezoresponse force microscopy, which is switchable and consistent with the previous results ${ }^{16}$.

For the PZT on the insulating STO substrate, a $180^{\circ}$ domain wall was observed in Supplementary Fig. 10, indicating the switchable nature. The mismatch between 
PZT and STO substrate is $\sim 1.2 \%$. Such compressive strain favours the out-of-plane domain orientations. The thickness of as-grown PZT film is $\sim 28 \mathrm{~nm}$. Cross-sectional TEM specimens were thinned to less than $30 \mu \mathrm{m}$ by mechanical polishing and followed by argon ion milling in a Precision Ion Polishing System 691 (Gatan). Ion milling procedure consists of two steps. In the first stage of coarse milling, the guns were at $4 \mathrm{keV}$ with angles $5^{\circ}$ and $-5^{\circ}$. In the following condition, the guns were set at $1 \mathrm{keV}$ for $5 \mathrm{~min}$ with angles of $3.5^{\circ}$ and $-3.5^{\circ}$, and further lowered to $0.1 \mathrm{keV}$ for $2 \mathrm{~min}$ for final surface cleaning. At the edges of the milled holes of the TEM specimen foils, the film thickness gradually changes from the full thickness to $0 \mathrm{~nm}$, forming a series of flat atomic terraces with typical width between 10 and 30-unit cells (see Supplementary Fig. 9). To study the thicknessdependent polarization, STEM images were acquired from different regions with different thicknesses. ABF images were recorded at $300 \mathrm{kV}$ in JEM ARM300CF (JEOL Ltd.). The convergence semi-angle for imaging is $24 \mathrm{mrad}$, collection semiangles snap is $12-24$ mrad for ABF imaging. All high-resolution ABF images used in this work were raw data without any post filtering. We deliberately choose those wider flat terraces and the middle of a single flat terrace for calculation to minimize the effects of the inhomogeneous thickness. Thus, the continuously changed thickness is not the main source of error in our case. The error bars of data points in Figs 2 and 3 mainly come from the (relatively random) spatial fluctuation of the structure in the TEM specimens.

STEM image analysis. Atom positions were determined by simultaneously fitting with two-dimensional Gaussian peaks to an a priori perovskite unit cell using a MatLab code. The $\mathrm{TiO}_{2}$ plane between the first $\mathrm{SrO}$ and $\mathrm{PbO}$ planes is defined as the interface and labelled as the $\# 0$ atomic layer. To define the surface layer $\# N$ for a certain region, all the atom columns in the $\# N$ layer should be visible and thus can be fitted with Gaussian peaks for distance calculation, and then $N$ is defined to be the thickness of the film. The lattice constant and bond-length for atomic columns in the \#n layer are the distance between the $\# n$ and $\#(n+1)$ layer, where $n \geq 2$. Taking the oxygen sublattice as the reference (that is, $\delta u_{\mathrm{O}}=0$ ), the displacements of $\mathrm{Pb}$ columns respective to the neighbouring $\mathrm{O}$ is $\delta u_{\mathrm{Pb}}=\frac{d_{1}-d_{2}}{2}$ and $\mathrm{Zr} / \mathrm{Ti}$ respective to the neighbouring $\mathrm{O}$ is $\delta u_{\mathrm{Zr} / \mathrm{Ti}}=\frac{d_{3}-d_{4}}{2}$, where $d_{1}, d_{2}^{2}$ are long and short $\mathrm{Pb}-\mathrm{O}$ bond length along $c$ direction respectively, and $d_{3}, d_{4}$ are short and long $\mathrm{Zr} / \mathrm{Ti}-\mathrm{O}$ bond length along $c$ direction respectively in Fig. 1c. The bond length $d_{i}(i=1,2,3$ and 4$)$ are calculated on the basis of the fitted atomic positions. The values of Born effective charges for cubic STO and tetragonal PTO were calculated from $a b$ initio theory ${ }^{35}$. Small displacements (a few picometres) can exist in the bulk substrate. Such small systematic-error-displacements and polarization originate from the tiny specimen mis-tilt (a few mrad) between optical axis and specimen ${ }^{42}$, which is unavoidable during experiments. These systematic-error-displacements are used as a reference for extracting the polarization in the ultrathin PZT films. The extracted polarization values in Figs 2 and 3 are calibrated by the references (that is, the mean of displacements in substrate/electrode bulk is set to be zero). The presence of point defects (for example, oxygen vacancies) would cause local structure distortion. Owing to the strong channelling effect along the atomic column, we can precisely determine the atomic column positions even including some point defects. For a specific atomic column, one oxygen vacancy may cause surrounding atom-position-shift. However, this shifted atom should not change the image contrast in the STEM image because the well-aligned atoms will dominate the total contrast due to the strong channelling effects. Therefore, the effects of point defects that cause localized structure distortion can be negligible in the STEM images.

Data availability. The data that support the findings of this study are available from the corresponding author upon request.

\section{References}

1. Lines, M. E. \& Glass, A. M. Principles and Applications of Ferroelectrics and Related Materials (Clarendon Press, 1977).

2. Shaw, T. M., Trolier-McKinstry, S. \& McIntyre, P. C. The properties of ferroelectric films at small dimensions. Annu. Rev. Mater. Sci. 30, 263-298 (2000)

3. Batra, I. P. \& Silverma, B. D. Thermodyanic stability of thin ferroelectric films. Solid State Commun. 11, 291-294 (1972).

4. Mehta, R. R., Silverma, B. D. \& Jacobs, J. T. Depolarization fields in thin ferroelectric films. J. Appl. Phys. 44, 3379-3385 (1973).

5. Tybell, T., Ahn, C. H. \& Triscone, J. M. Ferroelectricity in thin perovskite films. Appl. Phys. Lett. 75, 856-858 (1999).

6. Fong, D. D. et al. Ferroelectricity in ultrathin perovskite films. Science 304, 1650-1653 (2004)

7. Wen, Z. et al. Ferroelectric-field-effect-enhanced electroresistance in metal/ferroelectric/semiconductor tunnel junctions. Nat. Mater. 12, 617-621 (2013).

8. Tenne, D. A. et al. Ferroelectricity in Ultrathin $\mathrm{BaTiO}_{3}$ Films: Probing the Size Effect by Ultraviolet Raman Spectroscopy. Phys. Rev. Lett. 103, 177601 (2009).

9. Chu, Y. H. et al. Ferroelectric size effects in multiferroic $\mathrm{BiFeO}_{3}$ thin films. Appl. Phys. Lett. 90, 2750524 (2007).
10. Maksymovych, P. et al. Ultrathin limit and dead-layer effects in local polarization switching of $\mathrm{BiFeO}_{3}$. Phys. Rev. B 85, 014119 (2012).

11. Ghosez, P. \& Rabe, K. M. Microscopic model of ferroelectricity in stress-free $\mathrm{PbTiO}_{3}$ ultrathin films. Appl. Phys. Lett. 76, 2767-2769 (2000).

12. Junquera, J. \& Ghosez, P. Critical thickness for ferroelectricity in perovskite ultrathin films. Nature 422, 506-509 (2003).

13. Sai, N., Kolpak, A. M. \& Rappe, A. M. Ferroelectricity in ultrathin perovskite films. Phys. Rev. B 72, 020101 (2005).

14. Lichtensteiger, C. et al. Ferroelectricity and tetragonality in ultrathin $\mathrm{PbTiO}_{3}$ films. Phys. Rev. Lett. 94, 047603 (2005).

15. Kim, Y. S. et al. Critical thickness of ultrathin ferroelectric $\mathrm{BaTiO}_{3}$ films. Appl. Phys. Lett. 86, 1880443 (2005).

16. Nagarajan, V. et al. Size effects in ultrathin epitaxial ferroelectric heterostructures. Appl. Phys. Lett. 84, 5225-5227 (2004).

17. Bune, A. V. et al. Two-dimensional ferroelectric films. Nature 391, 874-877 (1998).

18. Lee, D. et al. Emergence of room-temperature ferroelectricity at reduced dimensions. Science 349, 1314-1317 (2015).

19. Streiffer, S. K. et al. Observation of nanoscale 180 degrees stripe domains in ferroelectric $\mathrm{PbTiO}_{3}$ thin films. Phys. Rev. Lett. 89, 067601 (2002).

20. Kornev, I., Fu, H. X. \& Bellaiche, L. Ultrathin films of ferroelectric solid solutions under a residual depolarizing field. Phys. Rev. Lett. 93, 196104 (2004)

21. Rault, J. E. et al. Thickness-Dependent Polarization of Strained BiFeO3 Films with Constant Tetragonality. Phys. Rev. Lett. 109, 267601 (2012).

22. Kim, D. J. et al. Polarization relaxation induced by a depolarization field in ultrathin ferroelectric $\mathrm{BaTiO}_{3}$ capacitors. Phys. Rev. Lett. 95, 237602 (2005).

23. Kretschmer, R. \& Binder, K. Surface effects on phase-transitions in ferroelectrics and dipolar magnets. Phys. Rev. B 20, 1065-1076 (1979).

24. Meyer, B. \& Vanderbilt, D. Ab initio study of $\mathrm{BaTiO}_{3}$ and $\mathrm{PbTiO}_{3}$ surfaces in external electric fields. Phys. Rev. B 63, 205426 (2001).

25. Gao, P. et al. Atomic mechanism of polarization-controlled surface reconstruction in ferroelectric thin films. Nat. Commun. 7, 11318 (2016).

26. Zembilgotov, A. G. et al. Ultrathin epitaxial ferroelectric films grown on compressive substrates: Competition between the surface and strain effects. J. Appl. Phys. 91, 2247-2254 (2002).

27. Ihlefeld, J. et al. Scaling Effects in Perovskite Ferroelectrics: Fundamental Limits and Process-Structure-Property Relations. J. Am. Ceram. Soc. 99, 2537-2557 (2016).

28. Gerra, G. et al. Ionic polarizability of conductive metal oxides and critical thickness for ferroelectricity in $\mathrm{BaTiO}_{3}$. Phys. Rev. Lett. 96, 107603 (2006).

29. Sepliarsky, M., Stachiotti, M. G. \& Migoni, R. L. Interface effects in ferroelectric $\mathrm{PbTiO}_{3}$ ultrathin films on a paraelectric substrate. Phys. Rev. Lett. 96, 137603 (2006).

30. Chisholm, M. F. et al. Atomic-Scale Compensation Phenomena at Polar Interfaces. Phys. Rev. Lett. 105, 197602 (2010).

31. Jia, C.-L. et al. Unit-cell scale mapping of ferroelectricity and tetragonality in epitaxial ultrathin ferroelectric films. Nat. Mater. 6, 64-69 (2007).

32. Duan, C. G. et al. Interface effect on ferroelectricity at the nanoscale. Nano Lett 6, 483-487 (2006).

33. Fong, D. D. et al. Stabilization of monodomain polarization in ultrathin $\mathrm{PbTiO}_{3}$ films. Phys. Rev. Lett. 96, 127601 (2006).

34. Findlay, S. D. et al. Robust atomic resolution imaging of light elements using scanning transmission electron microscopy. Appl. Phys. Lett. 95, 191913 (2009).

35. Zhong, W., Kingsmith, R. D. \& Vanderbilt, D. Giant LO-TO splitting sin perovskite ferroelectrics. Phys. Rev. Lett. 72, 3618-3621 (1994).

36. Cohen, R. E. Origin of ferroelectricity in perovskite oxides. Nature 358, 136-138 (1992).

37. Kalinin, S. V. et al. Surface Screening Mechanisms in Ferroelectric Thin Films and its Effect on Polarization Dynamics and Domain Structures. Preprint at http://arxiv.org/abs/1612.08266 (2016).

38. Tagantsev, A. K. et al. Ferroelectric materials for microwave tunable applications. J. Electroceram. 11, 5-66 (2003).

39. Stephenson, G. B. \& Highland, M. J. Equilibrium and stability of polarization in ultrathin ferroelectric films with ionic surface compensation. Phys. Rev. B 84, 064107 (2011).

40. Eliseev, E. A., Kalinin, S. V. \& Morozovska, A. N. Finite size effects in ferroelectric-semiconductor thin films under open-circuit electric boundary conditions. J. Appl. Phys. 117, 034102 (2015).

41. Polking, M. J. et al. Ferroelectric order in individual nanometre-scale crystals. Nat. Mater. 11, 700-709 (2012).

42. Gao, P. et al. Misalignment Induced Artifacts in Quantitative Annular BrightField Imaging. Microsc. Microanal. 22(Suppl 3): 888-889 (2016).

\section{Acknowledgements}

P.G. acknowledges the support from the National Basic Research Program of China (2016YFA0300804 and 2016YFA0300903), National Natural Science Foundation of China (51672007, 51502007, 51502032 and 51522201), the National Program for Thousand Young Talents of China and '2011 Program' Peking-Tsinghua-IOP 
Collaborative Innovation Centre for Quantum Matter. H.-J.L., Y.-L.H. and Y.-H.C. were supported by the Ministry of Science and Technology, R.O.C. (MOST 103-2119-M009-003-MY3), and Centre for Interdisciplinary Science of National Chiao Tung University. R.I., B.F., N.S. and Y.I. were supported in part by the Grant-in-Aid for Scientific Research on Innovative Areas "Nano Informatics" (Grant No. 25106003) from Japan Society for the Promotion of Science (JSPS). A part of this work was conducted at the Research Hub for Advanced Nano Characterization, The University of Tokyo, supported by "Nanotechnology Platform" (Project No. 12024046), sponsored by MEXT, Japan.

\section{Author contributions}

P.G. and Y.I. conceived and designed this work. H.-J.L. and Y.-L.H. grew thin films under direction of Y.-H.C. STEM experiments was carried out by P.G. and assisted by R.I. and B.F. The data analysis was mainly performed by Z.Z., M.L. under direction of P.G. and L.L. N.S., X.M., K.L., E.-G.W., D.Y. contributed data analysis and discussion. The manuscript was prepared by the P.G. All the authors commented the manuscript.

\section{Additional information}

Supplementary Information accompanies this paper at http://www.nature.com/ naturecommunications
Competing interests: The authors declare no competing financial interests.

Reprints and permission information is available online at http://npg.nature.com/ reprintsandpermissions/

How to cite this article: Gao, P. et al. Possible absence of critical thickness and size effect in ultrathin perovskite ferroelectric films. Nat. Commun. 8, 15549 doi: 10.1038/ncomms15549 (2017).

Publisher's note: Springer Nature remains neutral with regard to jurisdictional claims in published maps and institutional affiliations.

(c) (i) This work is licensed under a Creative Commons Attribution 4.0 International License. The images or other third party material in this article are included in the article's Creative Commons license, unless indicated otherwise in the credit line; if the material is not included under the Creative Commons license, users will need to obtain permission from the license holder to reproduce the material. To view a copy of this license, visit http://creativecommons.org/licenses/by/4.0/

(C) The Author(s) 2017 